EXTENDED REPORT

\title{
The expression of native and cultured human retinal pigment epithelial cells grown in different culture conditions
}

\author{
J Tian, K Ishibashi, S Honda, S A Boylan, L M Hjelmeland, J T Handa
}

Br J Ophthalmol 2005;89:1510-1517. doi: 10.1136/bjo.2005.072108

See end of article for authors' affiliations

Correspondence to: James T Handa, MD; 3-

109 Jefferson Street Building; Johns Hopkins Hospital; $600 \mathrm{~N}$ Wolfe Street; Baltimore, $M D$ 21287, USA; jthanda@ jhmi.edu

Accepted for publication 6 June 2005

\begin{abstract}
Aim: To determine the transcriptional proximity of retinal pigment epithelium (RPE) cells grown under different culture conditions and native RPE.

Methods: ARPE-19 cells were grown under five conditions in 10\% $\mathrm{CO}_{2}$ : "subconfluent" in DMEM/F12 + $10 \% \mathrm{FBS}$, "confluent" in serum and serum withdrawn, and "differentiated" for 2.5 months in serum and serum withdrawn medium. Native RPE was laser microdissected. Total RNA was extracted, reverse transcribed, and radiolabelled probes were hybridised to an array containing 5353 genes. Arrays were evaluated by hierarchical cluster analysis and significance analysis of microarrays.

Results: $78 \%$ of genes were expressed by native RPE while $45.3-47.7 \%$ were expressed by ARPE- 19 cells, depending on culture condition. While the most abundant genes were expressed by native and cultured cells, significant differences in low abundance genes were seen. Hierarchical cluster analysis showed that confluent and differentiated, serum withdrawn cultures clustered closest to native RPE, and that serum segregated cultured cells from native RPE. The number of differentially expressed genes and their function, and profile of expressed and unexpressed genes, demonstrate differences between native and cultured cells.

Conclusions: While ARPE-19 cells have significant value for studying RPE behaviour, investigators must be aware of how culture conditions can influence the mRNA phenotype of the cell.
\end{abstract}

$\mathrm{T}$ he retinal pigment epithelial (RPE) cell line ARPE-19, displays significant functional differentiation that mimics native RPE. ${ }^{1}$ It provides a dependable source of cultured RPE cells for study. At the time of writing, 121 PubMed publications have utilised this cell line. The transcriptional profile of RPE cultures including ARPE-19 cells, and its proximity to native RPE, is however, unknown.

Recently, we found transcriptional differences among ARPE-19 cells grown on different matrices. ${ }^{2}$ Unexpectedly, ARPE-19 cells grown on plastic displayed the closest phenotype to native RPE. While obvious that the transcriptome will be influenced by culture conditions, few studies are available to articulate these differences. We hypothesised that culture conditions could be optimised so ARPE-19 cells could simulate the native RPE mRNA phenotype. To accomplish this goal, we laser microdissected native RPE and compared its global transcriptional profile with ARPE-19 cells grown under different culture conditions.

\section{METHODS \\ Cell culture}

ARPE-19 cells were seeded at $10000 / \mathrm{cm}^{2}$ ("subconfluent" or "SS") for 3 days or $100000 / \mathrm{cm}^{2}$ in T-75 $\mathrm{cm}^{2}$ flasks and grown in Dulbecco's Modified Eagle medium/nutrient mixture F12 (DMEM/F12; BioWhittaker Inc, Walkersville, MD, USA) $+10 \%$ fetal bovine serum (FBS; UBI Upstate, Lake Placid, NY, USA) at $37^{\circ} \mathrm{C}$ in $10 \% \mathrm{CO}_{2}$. "Confluent" (CS) and "confluent, serum withdrawn" (CSW) cultures were grown for 7 days, and replaced with fresh medium containing serum or $1 \%$ bovine serum albumin (BSA), respectively for 3 more days. "Differentiated" (DS) and "differentiated serum withdrawn" (DSW) cells were grown for 2.5 months, and then in serum or $1 \%$ BSA for 3 days.

\section{Tissue preparation}

Ten eyes (45-95 years old) with a death enucleation time within 6 hours and from donors on life support systems for less than 24 hours were used since premorbid conditions have the greatest influence on RNA degradation ${ }^{3}$ (table 1).
Donors were free of ocular disease, systemic inflammatory disease, and diabetes mellitus. A macular calotte was dissected and cryoprotected as previously described..$^{45}$ Cryosections $(7 \mu \mathrm{m})$ were stained with haematoxylin and eosin Y (Fisher Scientific, Inc) before microdis`section.

\section{Laser capture microdissection}

RPE cells were removed with an Arcturus PixCell II (Arcturus Engineering, Inc Mountain View, CA, USA) as previously described. ${ }^{5}$ After dissection, the transfer cap was inspected for contaminating tissue before being placed in denaturing buffer.

\section{RNA extraction}

Total RNA was extracted using the RNeasy Mini-kit (Qiagen Inc, Valencia, CA, USA) and treated with DNase I (Qiagen, Inc) according to the manufacturer's recommendations. RNA quality was assessed by GAPDH expression using RT-qPCR with primers designed at the $5^{\prime}$ end of gene, and intact $28 \mathrm{~S}$ and $18 \mathrm{~S}$ ribosomal RNA bands of an RPE sample by gel electrophoresis.

\section{Probe synthesis}

Total RNA from 5000 cells was reverse transcribed with $50 \mu \mathrm{Ci}[33 \mathrm{P}] \mathrm{dCTP}$ and $50 \mu \mathrm{Ci}[33 \mathrm{P}] \mathrm{dATP}$ with $0.5 \mu \mathrm{g}$ oligo-dT according to our modified method of Sgroi et al. ${ }^{26} \mathrm{~A}$ second strand was synthesised with $50 \mu \mathrm{Ci}[33 \mathrm{P}] \mathrm{dCTP}$, $50 \mu \mathrm{Ci}[33 \mathrm{P}] \mathrm{dATP}, 500 \mathrm{ng}$ random hexamers, and $20 \mathrm{U}$ Klenow fragment (Gibco BRL). Probes were purified with a Bio-Spin 6 column (BioRad Laboratories, Hercules, CA, USA).

\section{Microarray analysis}

Labelled, double stranded cDNA was hybridised to the cDNA GeneFilter Human Microarray Release I (5353 genes;

Abbreviations: BSA, bovine serum albumin; CS, confluent serum; CSW, confluent, serum withdrawn; DMEM, Dulbecco's Modified Eagle medium; DS, differentiated serum; DSW, differentiated serum withdrawn; FBS, fetal bovine serum; FDR, false discovery rate; RPE, retinal pigment epithelium 


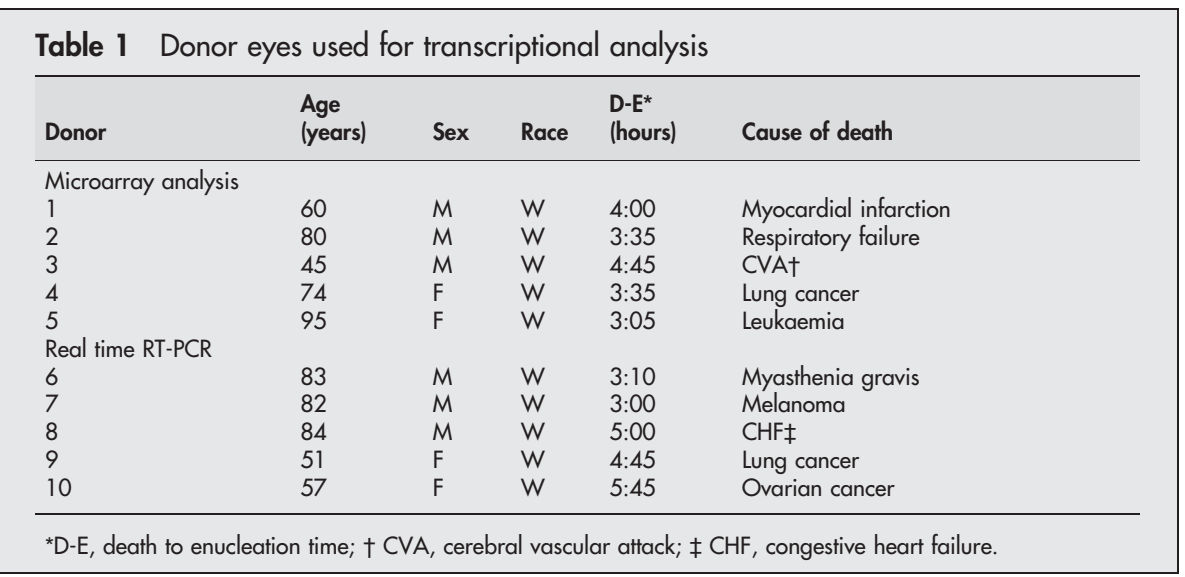

Invitrogen, Inc, Huntsville, AL, USA) using the manufacturer's protocol. This array contains an insert DNA from a sequence verified IMAGE/LLNL clone using the $3^{\prime}$ end of the gene. Arrays were exposed for 3 days to a high density phosphorimager screen (BioRad Laboratories) and scanned at $50 \mu \mathrm{m}$ resolution in a phosphorimager (FX Pro-Plus, BioRad Laboratories). The data appear on Gene Expression Omnibus (www.ncbi.nlm.nih.gov/geo/) using platform number GPL1488, and accession numbers GSM32029-GSM32048. Since Lee et al demonstrated that at least three replicate experiments are necessary for reliable microarray results, ${ }^{7}$ for each condition, at least three independent experiments were performed.

\section{Image and statistical analysis}

Signal intensity for each gene was quantified and normalised to $75 \%$ of the average signal intensity of the entire array by Pathways 3 software (Invitrogen, Inc). An individual gene was "expressed" if the signal intensity was $\geqslant 1.4$-fold above background $^{8}$ in at least two of three, and three of five experiments for cultured and native RPE, respectively.

Gene expression signals were scaled according to the method of Tusher et al. ${ }^{29}$ A reference set was generated using the average expression from all arrays. Each hybridisation signal was compared with the reference signal in a cube root scatter plot, and intensity values for each gene were corrected with a "scaling factor."

Hierarchical cluster analysis was performed with average linkage clustering by Cluster, and visualised with TreeView. ${ }^{10}$ Significance analysis of microarrays (SAM; version 1.12) determined differential gene expression between conditions. ${ }^{11}$

\section{Real time RT-PCR}

Total RNA (100 ng) was reverse transcribed with Sensiscript (Qiagen, Inc) as previously described. ${ }^{2}$ Primer sequences were designed to span consecutive exons using Primer 3 (Whitehead Institute/MIT, Cambridge, MA, USA). Sequences were verified using NCBI Unigene (table 2). First strand cDNA was amplified using the LightCycler (Roche Diagnostics, Nutley, NJ, USA) in a final volume of $20 \mu \mathrm{l}$ containing SYBR Green PCR Master Mix ( $10 \mu \mathrm{l}$; Qiagen, Inc, USA), primers $(0.5 \mu \mathrm{M}$ each), and $2 \mu \mathrm{l}$ DNA in $2.5 \mathrm{mM}$ $\mathrm{MgCl}_{2}$ The standard curve for the gene of interest consisted of PCR products $\left(1-10^{-6} \mathrm{pg}\right)$. PCR products were checked by melting point analysis and quantified using the second derivate maximum values calculated by the Light-Cycler analysis software. Expression was normalised to acidic ribosomal phosphoprotein expression. ${ }^{12}$ The Student's $t$ test was used to compare the differential gene expression between conditions.

\section{RESULTS}

\section{Expression profile of native and cultured RPE cells}

Figure 1A shows healthy macular RPE cells with cuboidal columnar epithelial morphology and normal Bruch's membrane. Figure 1B shows the cryosection after microdissection, and figure $\mathrm{IC}$ shows the microdissected cells adherent to the transfer cap. Figure 2 demonstrates spindly appearing "SS" cells (fig 2A) and regular "CS" cells (Fig 2B). "DS" cells had cobblestone morphology with melanin pigment (fig 2C).

Scatter plots of microarray analysis for pairwise comparison of native RPE from the five donors, and cultured cells showed reasonable reproducibility (data not shown; $\mathrm{R}^{2}=0.829-0.995$ for all comparisons). Using our expression criterion, the number of genes expressed by native RPE was $78 \%$ (4177 genes) of the array. The number of genes expressed by ARPE-19 cells ranged from 45.3\% (CS), 45.4\% (DS), $46.5 \%$ (SS), $47.2 \%$ (CSW), to $47.7 \%$ (DSW).

Table 2 Real time RT-PCR primers and conditions

\begin{tabular}{|c|c|c|c|c|c|c|c|}
\hline Gene name & $\begin{array}{l}\text { GenBank } \\
\text { Acc No }\end{array}$ & & Sequence & Location & $\begin{array}{l}\text { Size } \\
\text { (BP) }\end{array}$ & Cycles & $\begin{array}{l}\operatorname{Tm} \\
\text { (C) }\end{array}$ \\
\hline SEC13-like 1 (S cerevisiae) & AA496784 & $\begin{array}{l}\mathrm{F} \\
\mathrm{R}\end{array}$ & $\begin{array}{l}\text { CGTGTGTTCATTTGGACCTG } \\
\text { CCCTCTGTCACTGATGCTGA }\end{array}$ & $868-1103$ & 236 & 45 & 55 \\
\hline $\begin{array}{l}\text { Topoisomerase (DNA) } \\
\text { II } \alpha 170 \mathrm{kDa}\end{array}$ & AA504348 & $\begin{array}{l}\mathrm{F} \\
\mathrm{R}\end{array}$ & $\begin{array}{l}\text { TCCTGCCAAAACCAAGAATC } \\
\text { GTACAGATITGCCCGAGGA }\end{array}$ & $4494-4666$ & 173 & 45 & 55 \\
\hline $\begin{array}{l}\text { ATPase, } \mathrm{H}+\text { transporting, } \\
\text { lysosomal VO subunit a isoform } 1\end{array}$ & AA427472 & $\begin{array}{l}\mathrm{F} \\
\mathrm{R}\end{array}$ & $\begin{array}{l}\text { TGCCCTGCACTACATAGCAC } \\
\text { GGGGAAGATCTCAGGGTCTC }\end{array}$ & $3326-3514$ & 189 & 45 & 55 \\
\hline $\begin{array}{l}\text { Oxidase (cytochrome c) } \\
\text { assembly 1-like }\end{array}$ & AA598582 & $\begin{array}{l}\mathrm{F} \\
\mathrm{R}\end{array}$ & $\begin{array}{l}\text { GTCGAATCAGAGAGGCCAAG } \\
\text { GAGATCCTGGAACCACCAGA }\end{array}$ & $722-948$ & 227 & 45 & 55 \\
\hline $\begin{array}{l}\text { START domain containing } 4 \text {, } \\
\text { sterol regulated }\end{array}$ & H11369 & $\mathrm{F}$ & $\begin{array}{l}\text { ACCGCTCAAGGGGTTATTCT } \\
\text { CCAACACTITGGGAGGCTAA }\end{array}$ & $979-1158$ & 180 & 45 & 55 \\
\hline $\begin{array}{l}\text { Acidic ribosomal } \\
\text { phosphoprotein PO }\end{array}$ & M17885 & $\begin{array}{l}\mathrm{F} \\
\mathrm{R}\end{array}$ & $\begin{array}{l}\text { CGACCTGGAAGTCCAACTAC } \\
\text { ATCTGCTGCATCTGCTTG }\end{array}$ & $93-201$ & 109 & 45 & 53 \\
\hline
\end{tabular}



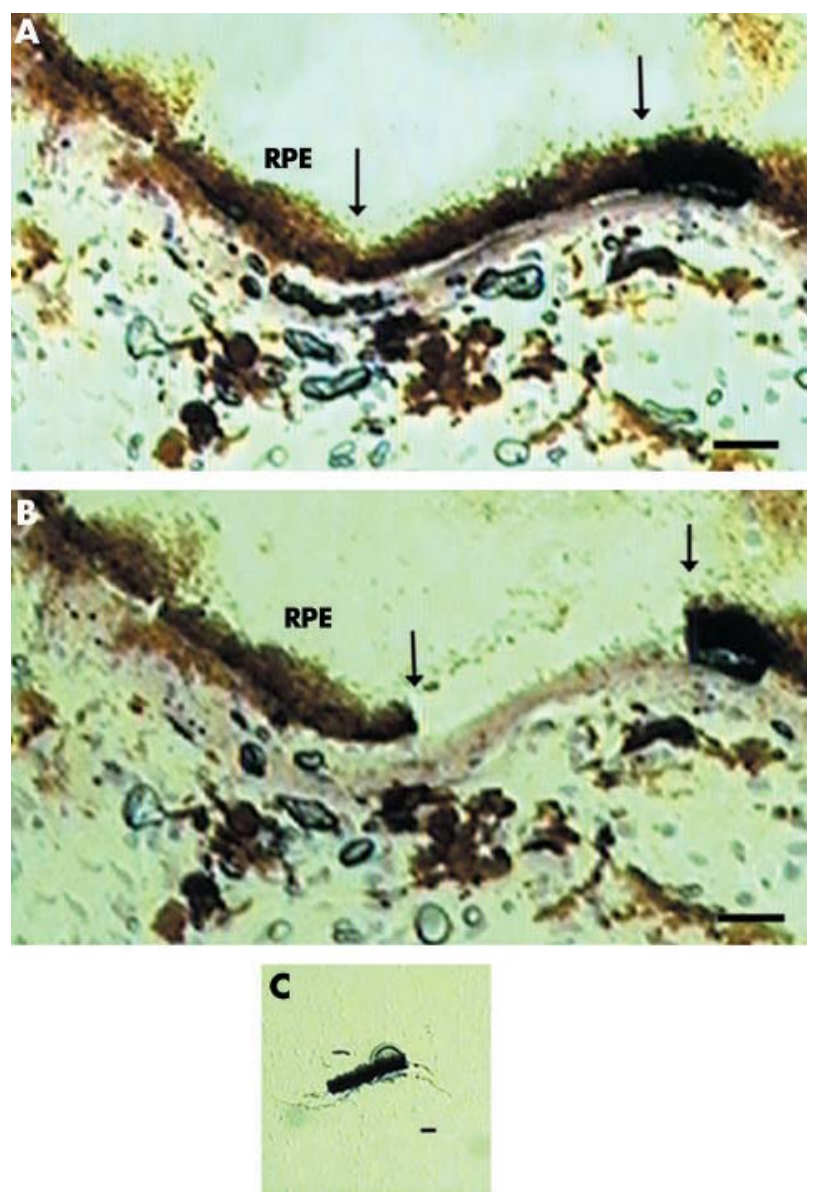

Figure 1 Photomicrograph of macular RPE/Bruch's membrane with normal morphology before (A) and after (B) laser capture microdissection, as described in the methods. Arrow indicates area of laser dissection. Five laser spots dissected five RPE cells, as seen on the transfer cap (C). Bar $=10 \mu \mathrm{m}$.

Table 3 shows the 50 most abundant genes from native RPE cells, of which 48 were also the 50 most abundantly expressed genes by ARPE-19 cells, regardless of culture condition (each separately analysed). While some genes illustrate the multiple functions of the RPE such as melanin biosynthesis (D-dopachrome tautomerase) or antioxidant function (Selenoprotein T), most genes have general cellular function such as protein processing (14\%), cytoskeleton $(8 \%)$, cell cycle $(8 \%)$, and differentiation $(6 \%)$. Two class unpaired SAM using a twofold expression differential showed no differentially expressed genes with any false discovery rate (FDR).

The majority of the 50 lowest abundance genes expressed by native RPE cells have function related to transcription factors (16\%), metabolism $(6 \%)$, or are unknown $(48 \%)$. However, a great number of genes had low expression. To further assess the proximity of low abundance genes between native and cultured RPE, 807 genes expressed by native RPE were identified by doubling the arbitrary expression units of the lowest 50 genes. The number of genes expressed by different culture conditions was low: SS (239 genes; $29.6 \%$ ), CSW (24l genes; $29.8 \%$ ), CS (223 genes; $27.6 \%$ ), DSW (263 genes, $32.4 \%$ ), DS (226 genes; $28.0 \%$ ).

\section{Cluster analysis of native and cultured RPE}

Unsupervised hierarchical cluster analysis showed that CSW or DSW cells clustered closest to native cells and were segregated from SS, CS, and DS cells (fig 3A). Since RPE cells
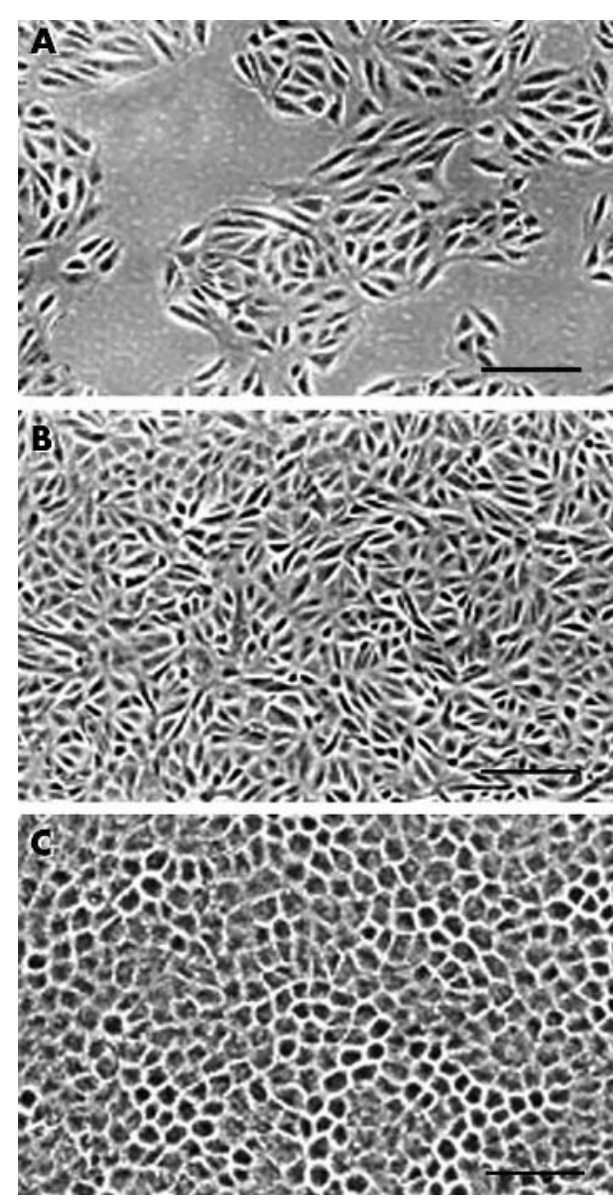

Figure 2 Phase contrast micrograph of ARPE-19 cells grown under various conditions, as outlined in the methods. (A) Subconfluent cells grown in DMEM/F12 + 10\% FBS; (B) confluent cells grown in DMEM/ $\mathrm{F} 12+10 \% \mathrm{FBS}$; and (C) differentiated cells grown for 2.5 months in DMEM/F12+10\% FBS. Bar $=50 \mu \mathrm{m}$.

undergo apoptosis and morphological deterioration with ageing, we identified 713 genes involved with differentiation or cell cycle/apoptosis. Supervised cluster analysis was similar to unsupervised cluster analysis (fig 3B).

\section{Differential expression of native and cultured RPE cells}

The expression profiles of CSW and SS cells were used to assess their proximity to native RPE since they were similar and dissimilar, respectively, to native RPE by cluster analysis. Firstly, CSW and SS cultures expressed only $60.5 \%$ and $59.6 \%$ of the genes, respectively, that were expressed by native RPE. Of the differentially expressed genes identified by SAM (FDR $7 \%)$ between native RPE and SS ( $\mathrm{n}=592$ genes), and native RPE and CSW ( $\mathrm{n}=318$ genes), 250 differentially expressed genes were in common, or $42 \%$ of SS and $78 \%$ of CSW genes, respectively. Of these differentially expressed genes, $36 \%$ had no reported function while $7.2 \%$ were related to cell cycle/ apoptosis, $4.4 \%$ to cell structure, $5.6 \%$ to metabolism, $7.6 \%$ to protein processing, $8.4 \%$ to signal transduction, and $6.8 \%$ to transcriptional regulation.

Secondly, of 1175 genes unexpressed by native RPE, 324 $(27.6 \%)$ of these genes were expressed by CSW cells. The function of genes expressed by CSW cells include signal transduction $(7.7 \%)$, cell cycle/apoptosis $(7.7 \%)$, transcriptional regulation $(7.1 \%)$, protein processing $(6.5 \%)$, intracellular transport (4\%), cell adhesion $(3.3 \%)$, cytoskeleton $(1.5 \%)$, and unknown function $(27.8 \%)$. Of genes not 
Table 350 Most abundant genes expressed by native macular RPE cells

\begin{tabular}{|c|c|c|c|}
\hline Gene name & $\begin{array}{l}\text { GenBank } \\
\text { Acc No }\end{array}$ & $\begin{array}{l}\text { Signal } \\
(\mathrm{AU})^{*}\end{array}$ & Biological function \\
\hline Cut-like 1, CCAAT displacement protein (Drosophila) & AA284408 & 49.2 & Development \\
\hline cDNA FU34046 fis, clone FCBBF2007610 & W15465 & 44.7 & Unknown \\
\hline Solute carrier family 6 (neurotransmitter transporter, betaine/GABA), member 12 & N49856 & 37.7 & Neurotransmitter \\
\hline Nephronophthisis 3 (adolescent) & Н93118 & 36.3 & Unknown \\
\hline Junctional adhesion molecule 2 & R68464 & 36.2 & Cell adhesion \\
\hline Prefoldin 4 & AA253430 & 35.9 & Protein folding \\
\hline EST & R32754 & 34.1 & Unknown \\
\hline Selenoprotein T & R78516 & 33.5 & Antioxidant \\
\hline Ubiquitin conjugating enzyme E2I (UBC9 homologue, yeast) & AA487197 & 33.1 & Protein degradation \\
\hline Biogenesis of lysosome related organelles complex-1, subunit 1 & H94857 & 32.2 & Unknown \\
\hline$C D C 28$ protein kinase regulatory subunit $1 \mathrm{~B}$ & AA459292 & 31.4 & Cell cycle \\
\hline D-dopachrome tautomerase mRNA, complete cds & AA292995 & 31.0 & Unknown \\
\hline Zinc finger protein 258 & AA280676 & 29.4 & Development \\
\hline Endothelin converting enzyme 1 & AA279429 & 28.6 & Cell-cell signalling \\
\hline Multiple endocrine neoplasia I & AA261796 & 27.6 & Transcription regulation \\
\hline CDC28 protein kinase regulatory subunit 2 & AA397813 & 27.0 & Cell cycle \\
\hline Interleukin 24 & AA281635 & 26.5 & Immune response, apoptosis \\
\hline EST & N92646 & 26.0 & Unknown \\
\hline Ubiquinol-cytochrome c reductase, Rieske iron-sulfur polypeptide 1 & AA448184 & 24.9 & electron transport \\
\hline Profilin 1 & AA521431 & 23.5 & Cell structure \\
\hline $\mathrm{BCL} 2$ related protein $\mathrm{A} 1$ & AA459263 & 23.1 & Apoptosis inhibition \\
\hline EST & R76499 & 23.1 & Unknown \\
\hline Kinesin family member $5 B$ & AA046690 & 21.9 & Organelle transport \\
\hline Rho GTPase activating protein $1 \dagger$ & AA443506 & 21.5 & Cell structure \\
\hline Adaptor related protein complex $1, \gamma 1$ subunit & W07300 & 21.5 & Protein sorting \\
\hline NudE nuclear distribution gene $\mathrm{E}$ homologue-like 1 (A. nidulans) & R94775 & 21.1 & Unknown \\
\hline EST & H93842 & 20.8 & Unknown \\
\hline Sarcolipin & AA196465 & 20.7 & Intracellular transport \\
\hline Myeloid cell nuclear differentiation antigen & N29376 & 20.7 & Transcription regulation \\
\hline PDGFA associated protein $1 \dagger$ & AA490300 & 20.6 & Cell cycle \\
\hline Guanine nucleotide binding protein ( $G$ protein), $\alpha$ inhibiting activity polypeptide 3 & AA490256 & 20.3 & Unknown \\
\hline Chitinase 3-like 1 (cartilage glycoprotein-39) & AA434115 & 20.2 & Metabolism \\
\hline EST & H93906 & 20.1 & Unknown \\
\hline Chromosome 14 open reading frame 2 & T90621 & 20.1 & Unknown \\
\hline Ataxin 2 related protein & AA029963 & 19.9 & Unknown \\
\hline Zinc finger protein 258 & AA280677 & 19.9 & Development \\
\hline DnaJ (Hsp40) homolog, subfamily B, member 1 & AA481758 & 19.8 & Protein folding \\
\hline Vesicle docking protein pl 15 & AA504342 & 19.5 & $\begin{array}{l}\text { Vesicle docking during } \\
\text { exocytosis }\end{array}$ \\
\hline Secretory carrier membrane protein 2 & R32802 & 19.4 & Protein transport \\
\hline Cas- $\mathrm{Br}-\mathrm{M}$ (murine) ecotropic retroviral transforming sequence & N94234 & 19.4 & Cell growth \\
\hline EST & T60223 & 19.3 & Unknown \\
\hline Chromosome $\mathrm{X}$ open reading frame 12 & AA455272 & 18.8 & Unknown \\
\hline EST & R69566 & 18.8 & Unknown \\
\hline Decay accelerating factor for complement (CD55, Cromer blood group system) & R09561 & 18.6 & Complement pathway \\
\hline CTF8, chromosome transmission fidelity factor 8 homologue ( $S$ cerevisiae) & N57731 & 18.5 & Unknown \\
\hline EST & R25153 & 18.5 & Unknown \\
\hline Villin 2 (ezrin) & AA411440 & 18.5 & Cell structure \\
\hline Ankyrin repeat domain 1 (cardiac muscle) & AA488072 & 18.5 & Defence response \\
\hline Cytoskeleton associated protein 1 & AA504554 & 18.5 & Cell structure \\
\hline EST & W44701 & 18.3 & Unknown \\
\hline
\end{tabular}

expressed by native RPE, 349 genes (29.7\%) were expressed by SS cells. The most common function of genes expressed by SS cells included cell cycle/apoptosis $(9.7 \%)$, protein processing $(7.7 \%)$, signal transduction $(6.9 \%)$, transcription regulation $(6.3 \%)$, small molecule transport $(4.9 \%)$, cell adhesion $(4.3 \%)$, immune response $(3.4 \%)$, RNA processing $(2.3 \%)$, and unknown function $(28.9 \%)$.

The number of differentially expressed genes between native and ARPE-19 cells was used as a third assessment of transcriptional proximity. An FDR $<10 \%$ is a reliable indicator of statistical precision for studies using human tissue. ${ }^{13}$ Using two class unpaired SAM analysis between native RPE and CSW (FDR 7.2\%) or SS cells (FDR 7.4\%), 317 and 591 differentially expressed genes respectively, were identified.

The function of differentially expressed genes between native RPE and individual culture conditions was a fourth factor used to evaluate the similarity of expression profiles. The functional annotation of the 317 differentially expressed genes between native RPE and CSW cells included cell cycle/ apoptosis $(10.7 \%)$, protein processing $(6.9 \%)$, signal transduction $(5.7 \%)$ and transcription factors $(5.7 \%)$, metabolism (6.3\%), and unknown (42.7\%). Analysis using highly stringent conditions (FDR 1.5\%) showed that 48 genes were underexpressed and five genes were overexpressed by native RPE compared to CSW cells (table 4). Of this gene set, $21 \%$ were related to protein processing.

The biological function of the 591 differentially expressed genes between native and SS cells included cell cycle/ apoptosis $(9.6 \%)$, protein processing $(8.8 \%)$, metabolism $(8.3 \%)$, signal transduction $(7.4 \%)$, transcriptional regulation (5.9\%), cytoskeleton (4.7\%), cell adhesion (3.4\%), and unknown (35.4\%). Analysis using highly stringent conditions (FDR $1.4 \%$ ) identified 41 genes that were underexpressed and 5 genes that were overexpressed by native RPE compared to SS cells (table 5). Again, protein processing genes comprised $17 \%$ of differentially expressed genes. 
A

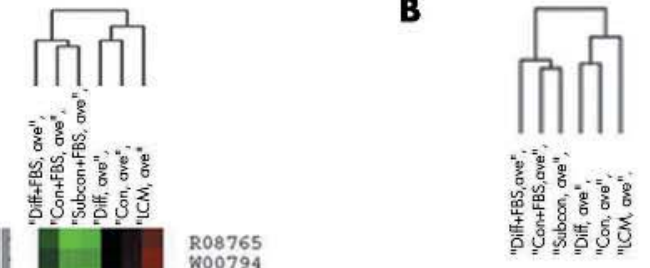

R99526

R07295
T95185

T95185
R62242

R62242

RA1765366

R27711

R.00594
AR488336

AA4 88336
AA2 33549

A.2 233549
R98278

R98278
N67017

R02663

H97765

N91952

N59482

W30988

W32403

T66956

H94236

AA47852

R.21261

$\mathrm{H} 94903$
$\mathrm{R} 40324$

$\mathrm{R} 40324$
$\mathrm{R} 83270$

$R 83270$
$H 95086$

H
R850843

$R 86843$
$H 05800$

H05800
AA2 280691

AA2 80691
R99573

R99573
R02800

R02800
W74565

W74565
R33154

N32146

R 92352

AA504682

AA485523

AA1 9255

W96250

R38682

N51278

R64153

N73201

R33011

W37306

W37306

W58092

N73227
AA451969

AA451969

AA4 53293

AA280214

AA280214

พ02483

H48420

R22523

AA479102

AA458661

AA12955:

R54818

N36882

AA447531

AA4 23867

A 405885

A 52138

AA5 2138

R67099

N72313

R32875
T 97592

N49436

N49436
R94893

AA521466

N66001

A44 49430

R24356

H74101

AA42845

R43325

R78539

R23222

W01 905

AA0 25937

AA019316

T69477

N53136

W47576
T53626

T5 3626
T55639

T55639
R71531

B64601

H64601
AA424516

AA42451
R16656

AA1 13331

AA028987

R33355

R01279
Serum influences the expression of cultured RPE cells Since serum divided the culture conditions, and native RPE clustered with serum withdrawn conditions, two class unpaired SAM analysis (FDR 9.9\%) between serum and serum withdrawn conditions identified 317 differentially expressed genes. Cells that were grown with serum removed had 207 genes overexpressed and 110 genes underexpressed compared to cells grown in serum. The biological function of differentially expressed genes included protein processing $(11.7 \%)$, signal transduction (11.7\%), cell cycle (5.4\%), metabolism $(5.4 \%)$, transcription factors $(4.7 \%)$, cell adhesion $(2.8 \%)$, cell structure $(1.8 \%)$, and unknown $(38.8 \%)$. The actual differential expression was small, ranging from 15$30 \%$. To evaluate the influence of genes with larger differential expression, SAM analysis with more stringent FDR $(4.6 \%)$ and a twofold differential expression threshold was performed (table 6). Three apoptosis, and three differentiation genes were identified.

\section{Real time RT-qPCR validation}

Real time RT-qPCR was performed on cells grown under identical culture conditions, but separate experiments from the microarray analysis, and native RPE from five different globes to expand the differential expression pattern beyond the eyes used for microarray experiments. Since the purpose of this investigation was to evaluate global gene expression changes, genes were randomly selected. Table 7 shows that all five genes had similar expression patterns as observed on the arrays.

\section{DISCUSSION}

We showed previously that confluent ARPE-19 cells grown on different matrices had different transcriptional profiles from native RPE, and that cells grown on plastic had the closest transcriptome to native RPE. ${ }^{2}$ To improve the transcriptional proximity of ARPE-19 cells grown on plastic to native RPE, we varied the culture conditions and evaluated global expression trends, a more informative benchmark than individual gene expression. With this end point, CSW and DSW cells were most similar to native RPE. While each culture condition preserved the expression of the most abundant genes, significant transcriptional differences exist between native and cultured cells including the number of genes on the array that were expressed and not expressed, the number and function of differentially expressed genes, and the number of expressed low abundance genes. Our results suggest that the global expression profiles can be improved by varying the culture conditions, but significant widespread differences remain between cultured and native RPE.

With ageing, the RPE undergoes apoptosis and morphological deterioration. Supervised cluster analysis of 713 genes related to differentiation or cell cycle/apoptosis was similar to unsupervised analysis, further suggesting that CSW or DSW cells are most similar to native RPE. Further analysis of the transcriptome of CSW cells however, showed significant differences from native RPE including the number of genes

Figure 3 A cluster node of laser capture microdissected RPE cells and ARPE-19 cells grown under five culture conditions. The similar dendrite length indicates relative similarity between conditions. (A) Unsupervised cluster analysis; (B) supervised cluster analysis. Red is $\log _{2}$ intensity value $<1$, green is $\log _{2}$ intensity value $>1$ after transformation, black is $\log _{2}$ intensity value $=1$, and grey indicates not expressed. "Diff+FBS" is differentiated ARPE-19 cells grown in serum; "Native" is laser captured RPE cells; "Subcon + FBS" is subconfluent ARPE-19 grown in serum; "Con+FBS" is confluent ARPE-19 cells grown in serum; "Con" is confluent serum withdrawn ARPE-19 cells; and "Diff" is differentiated serum withdrawn ARPE-19 cells. Individual genes are designated by ACC number. 
Table 4 Differential gene expression between confluent, serum withdrawn cultured and native RPE cells sorted by SAM (FDR $1.5 \%)$. Genes are sorted by biological function

\begin{tabular}{|c|c|c|c|c|}
\hline Gene name & GenBank Acc No & Score (d) & Fold change* & Biological function \\
\hline \multicolumn{5}{|l|}{48 Genes overexpressed by confluent serum withdrawn RPE } \\
\hline Lectin, galactoside binding, soluble, 9 (galectin 9) & AA434102 & 4.6899019 & NAt & Cell adhesion \\
\hline Cyclin dependent kinase 4 & AA486312 & 4.0367285 & NA & Cell cycle \\
\hline Pospholipase $\mathrm{A} 2$ receptor $1,180 \mathrm{kDa}$ & R91516 & 3.9299213 & NA & Cell cycle \\
\hline Integrin $\beta 1$ binding protein 1 & AA456882 & 3.8089025 & NA & Cell-matrix adhesion \\
\hline Integrin, $\alpha 2$ (CD49B, $\alpha 2$ subunit of VLA-2 receptor) & AA463610 & 3.7711619 & NA & Cell-matrix adhesion \\
\hline Actin, $\beta$ & R44290 & 4.0403841 & NA & Cytoskeleton \\
\hline Caldesmon 1 & AA076063 & 3.7303184 & 1.2 & Cytoskeleton; muscle contraction \\
\hline Oxidase (cytochrome c) assembly 1-like & AA598582 & 3.4692439 & 10.5 & Electron transport \\
\hline Tumour suppressor candidate 3 & N66008 & 5.2150083 & NA & Electron transport \\
\hline Cytochrome $\mathrm{c}$ oxidase subunit Vlc & AA456931 & 4.3479318 & NA & Electron transport \\
\hline Reproduction 8 & AA465570 & 5.736559 & NA & Fertilisation \\
\hline Sialyltransferase 1 ( $\beta$ galactoside $\alpha-2,6$-sialyltransferase) & AA598652 & 4.0207485 & NA & Immune response \\
\hline Epstein-Barr virus induced gene 3 & AA425028 & 3.4711393 & NA & Immune response \\
\hline Tumour necrosis factor receptor superfamily, member 5 & H98636 & 3.3610776 & NA & Immune response; apoptosis \\
\hline $\begin{array}{l}\text { Fucosyltransferase } 4(\alpha(1,3) \text { fucosyltransferase, myeloid } \\
\text { specific) }\end{array}$ & R28447 & 3.8948373 & NA & $\begin{array}{l}\text { Metabolism; carbohydrate } \\
\text { metabolism }\end{array}$ \\
\hline Histidine ammonia-lyase & W86776 & 4.2938508 & NA & $\begin{array}{l}\text { Metabolism; histidine } \\
\text { catabolism }\end{array}$ \\
\hline Epoxide hydrolase 2, cytoplasmic & R73525 & 5.1584747 & NA & $\begin{array}{l}\text { Metabolism; xenobiotic } \\
\text { metabolism }\end{array}$ \\
\hline Protein phosphatase 1, catalytic subunit, $\alpha$ isoform & AA443982 & 3.7866788 & NA & $\begin{array}{l}\text { Metabolism; glycogen } \\
\text { metabolism }\end{array}$ \\
\hline CAZ associated structural protein & R52873 & 3.3870227 & NA & Protein degradation \\
\hline Ubiquitin protein ligase E3C & AA284599 & 4.808889 & NA & Protein degradation \\
\hline $\begin{array}{l}\text { Ubiquitin conjugating enzyme E2D } 3 \text { (UBC4/5 homologue, } \\
\text { yeast) }\end{array}$ & AA017199 & 4.1638422 & NA & Protein degradation \\
\hline Proteasome regulatory particle subunit p $44 \mathrm{~S} 10$ & AA424807 & 3.8497784 & NA & Protein degradation \\
\hline HECT type E3 ubiquitin ligase & R87212 & 3.2685684 & NA & Protein degradation \\
\hline ATPase, $\mathrm{H}+$ transporting, lysosomal $\mathrm{VO}$ subunit a isoform $\mathrm{l}$ & AA427472 & 16.602038 & 2.8 & $\begin{array}{l}\text { Protein degradation; } \\
\text { acidification of organelles }\end{array}$ \\
\hline Protein-O-mannosyltransferase 1 & R13777 & 3.9364101 & NA & Protein modification \\
\hline Aspartylglucosaminidase & R42153 & 3.4922915 & NA & Protein modification \\
\hline Adaptor related protein complex $3, \beta 2$ subunit & H11692 & 4.1620878 & NA & Protein transport \\
\hline SEC13-like 1 (S. cerevisiae) & AA496784 & 4.1282042 & NA & Protein transport \\
\hline$\alpha$-2-macroglobulin & H06516 & 3.6385137 & NA & Protein transport \\
\hline RAEl RNA export 1 homolog ( $S$ pombe) & AA504128 & 3.4039961 & NA & RNA transport \\
\hline Protein tyrosine phosphatase, non-receptor type 1 & R06605 & 3.5456862 & NA & Signal transduction \\
\hline Zinc finger protein 593 & AA033532 & 3.3121002 & NA & Transcription regulation \\
\hline $\begin{array}{l}\text { Signal transducer and activator of transcription } 3 \text { interacting } \\
\text { protein } 1\end{array}$ & N77731 & 3.2713342 & NA & Transcription regulation \\
\hline Zinc finger protein 161 & AA232647 & 6.8052672 & 1.5 & $\begin{array}{l}\text { Transcription regulation; cellular } \\
\text { defense }\end{array}$ \\
\hline Topoisomerase (DNA) II alpha 170 kDa & AA504348 & 45.484875 & 17.4 & $\begin{array}{l}\text { Transcription regulation; DNA } \\
\text { topology }\end{array}$ \\
\hline Synaptoporin & H98620 & 4.1875559 & NA & Transport \\
\hline ATP binding cassette, subfamily $B$ (MDR/TAP), member 10 & R83875 & 3.3779001 & NA & Transport \\
\hline (1) & H66616 & 5.2260079 & NA & Unknown \\
\hline HIV-1 rev binding protein 2 & W52273 & 4.6580643 & 2.6 & Unknown \\
\hline EST & R73909 & 4.2685967 & NA & Unknown \\
\hline EST & AA411554 & 4.1332 & 1.5 & Unknown \\
\hline EST & R02609 & 3.9193518 & 1.5 & Unknown \\
\hline Cisplatin resistance associated & W77812 & 3.8728312 & NA & Unknown \\
\hline Reticulocalbin 1, EF-hand calcium binding domain & AA457719 & 3.3784786 & NA & Unknown \\
\hline Family with sequence similarity 13 , member $\mathrm{Al}$ & N51424 & 3.3669014 & NA & Unknown \\
\hline $\begin{array}{l}\text { Transcribed sequence with weak similarity to protein } \\
\text { ref:NP_060219.1 (H sapiens) hypothetical protein FL20294 } \\
\text { (H sapiens) }\end{array}$ & R51835 & 3.3217265 & 10.3 & Unknown \\
\hline $\begin{array}{l}\text { Similar to non-histone chromosomal protein HMG-14 (high } \\
\text { mobility group nucleosome binding domain 1) (LOC400452) mRNA }\end{array}$ & R53889 & 3.315474 & NA & Unknown \\
\hline EST & W19429 & 3.2800179 & NA & Unknown \\
\hline 5 genes underexpressed by confluent serum withdrawn RPE & & & & \\
\hline Phosphofructokinase, platelet & R38433 & -5.491407 & -2.0 & Metabolism; glycolysis \\
\hline $\begin{array}{l}\text { Mannosyl ( } \alpha-1,6-) \text {-glycoprotein } \beta-1,2-\mathrm{N} \text { - } \\
\text { acetylglucosaminyltransferase }\end{array}$ & AA485653 & -3.719321 & -2.0 & $\begin{array}{l}\text { Metabolism; oligosaccaride } \\
\text { synthesis }\end{array}$ \\
\hline START domain containing 4 , sterol regulated & $\mathrm{H} 11369$ & -3.790357 & -2.6 & $\begin{array}{l}\text { Metabolism; cholesterol } \\
\text { transporter }\end{array}$ \\
\hline Snail homologue 2 (Drosophila) & H57309 & -3.733474 & -6.3 & Transcription regulation \\
\hline CDNA FU42496 fis, clone BRACE2035003 & AA410207 & -4.944185 & -12.2 & Unknown \\
\hline
\end{tabular}

expressed and not expressed, and differentially expressed genes with a diversity of function. These data collectively suggest that while clustering closest to native RPE, the global transcriptional profile of CSW cells displayed multiple mRNA phenotypic differences which may influence ageing studies.
Culture conditions were segregated by serum. The function of differentially expressed genes, regardless of culture density, was quite wide ranging, which suggests that serum influences multiple cellular functions including protein processing, signal transduction, cell cycle, metabolism, and 
Table 5 Differential gene expression between subconfluent cultured and native RPE cells sorted by SAM (FDR 1.4\%). Genes are sorted by biological function

\begin{tabular}{|c|c|c|c|c|}
\hline Gene name & GenBank Acc No & Score (d) & Fold change $^{*}$ & Biological function \\
\hline \multicolumn{5}{|l|}{41 Genes overexpressed by subconfluent RPE } \\
\hline Collagen, type XVI, $\alpha 1$ & R54778 & 6.2086958 & $\mathrm{NA} \dagger$ & Cell adhesion \\
\hline Integrin, $\alpha 2$ (CD49B, $\alpha 2$ subunit of VLA-2 receptor & AA463610 & 5.9930352 & NA & Cell adhesion \\
\hline Thrombospondin 2 & $\mathrm{H} 38240$ & 5.5952514 & NA & Cell adhesion \\
\hline Insulin-like growth factor binding protein 3 & AA598601 & 6.4324886 & NA & Cell cycle \\
\hline Cyclin DI (PRAD1: parathyroid adenomatosis 1) & AA487700 & 5.9964532 & NA & Cell cycle \\
\hline $\begin{array}{l}\text { v-yes-1 Yamaguchi sarcoma viral related oncogene } \\
\text { homologue }\end{array}$ & R83836 & 9.0139898 & NA & Cell cycle \\
\hline Gap junction protein, $\beta 2,26 \mathrm{kDa}$ (connexin 26) & AA490688 & 5.8023088 & NA & Cell-cell signaling \\
\hline Actin, $\beta$ & R44290 & 9.7128396 & NA & Cytoskeleton \\
\hline Caldesmon 1 & AA076063 & 7.83121 & 1.4 & $\begin{array}{l}\text { Cytoskeleton; Muscle } \\
\text { contraction }\end{array}$ \\
\hline Reproduction 8 & AA465570 & 6.971362 & NA & Fertilisation \\
\hline $\begin{array}{l}\text { Tumour necrosis factor receptor superfamily, member } \\
25\end{array}$ & W76376 & 6.1518795 & NA & Immune response, apoptosis \\
\hline $\begin{array}{l}\text { Transcription factor } 12 \text { (HTF4, helix-loop-helix } \\
\text { transcription factors } 4 \text { ) }\end{array}$ & AA488497 & 7.1312589 & NA & $\begin{array}{l}\text { Immune response; } \\
\text { development }\end{array}$ \\
\hline $\begin{array}{l}\text { Glutamate receptor, ionotropic, } \mathrm{N} \text {-methyl D-aspartate } \\
2 \mathrm{~A}\end{array}$ & H08933 & 5.6530117 & NA & lon transport \\
\hline $\begin{array}{l}\text { Fucosyltransferase } 4(\alpha(1,3) \text { fucosyltransferase, } \\
\text { myeloid specific) }\end{array}$ & R28447 & 5.7747679 & NA & Metabolism, carbohydrate \\
\hline Microsomal glutathione S-transferase 1 & AA495936 & 6.6792779 & NA & Oxidative stress response \\
\hline HECT type E3 ubiquitin ligase & R87212 & 7.9988818 & NA & Protein degradation \\
\hline CAZ associated structural protein & R52873 & 5.6053736 & NA & Protein degradation \\
\hline 26 serine protease & $\mathrm{H} 04028$ & 7.4968725 & NA & $\begin{array}{l}\text { Protein degradation; } \\
\text { protease }\end{array}$ \\
\hline Elastase $3 \mathrm{~B}$, pancreatic & W40123 & 6.9857814 & NA & $\begin{array}{l}\text { Protein degradation; } \\
\text { protease }\end{array}$ \\
\hline Aspartylglucosaminidase & R42153 & 8.4918723 & NA & Protein modification \\
\hline SEC13-like 1 (S cerevisiae) & AA496784 & 10.424023 & NA & Protein transport \\
\hline Adaptor related protein complex $3, \beta 2$ subunit & $\mathrm{H} 11692$ & 5.6233762 & NA & Protein transport \\
\hline Peroxisome biogenesis factor 1 & AA427472 & 16.184002 & 2.4 & Proton transport \\
\hline $\begin{array}{l}\text { Splicing factor proline/glutamine rich (polypyrimidine } \\
\text { tract binding protein associated) }\end{array}$ & AA425853 & 7.7310373 & NA & RNA processing \\
\hline Tyrosine kinase 2 & AA482128 & 7.5206556 & NA & Signal transduction \\
\hline Inositol 1,4,5-triphosphate receptor, type 2 & AA479093 & 7.3995941 & NA & Signal transduction \\
\hline Protein phosphatase 1, catalytic subunit, aisoform & AA443982 & 6.5780078 & NA & Signal transduction \\
\hline Zinc finger protein 161 & AA232647 & 9.8923477 & 1.6 & $\begin{array}{l}\text { Transcription regulation; } \\
\text { defense response }\end{array}$ \\
\hline Topoisomerase (DNA) II $\beta 170 \mathrm{kDa}$ & AA504348 & 39.16232 & 16.9 & $\begin{array}{l}\text { Transcription regulation; } \\
\text { DNA topology }\end{array}$ \\
\hline Synaptoporin & H9862O & 10.04159 & NA & Transport \\
\hline $\begin{array}{l}\text { Guanine nucleotide binding protein (G protein), } \alpha \\
\text { inhibiting activity polypeptide } 3\end{array}$ & AA490256 & 5.6303528 & 1.5 & Transport \\
\hline $\begin{array}{l}\text { ATP binding cassette, subfamily B (MDR/TAP), } \\
\text { member } 10\end{array}$ & R83875 & 5.5977134 & NA & Transport \\
\hline $\begin{array}{l}\text { Human neuropeptide Y receptor Y1 (NPYY1) mRNA, } \\
\text { exon 2-3 and complete cds. }\end{array}$ & R19478 & 13.345719 & NA & Unknown \\
\hline $\begin{array}{l}\text { Syndecan } 2 \text { (heparan sulfate proteoglycan 1, cell } \\
\text { surface associated, fibroglycan) }\end{array}$ & H64346 & 9.7652404 & NA & Unknown \\
\hline Integral membrane protein $2 \mathrm{C}$ & AA034213 & 7.76812 & NA & Unknown \\
\hline Cisplatin resistance associated & W77812 & 7.6011771 & NA & Unknown \\
\hline IMAAGE:363103 & AA019511 & 7.0814745 & NA & Unknown \\
\hline IMAGE:327676 & W23757 & 6.326244 & NA & Unknown \\
\hline IMAGE:28309 & R40460 & 6.3181421 & 1.5 & Unknown \\
\hline IMAGE: 197888 & R96220 & 6.1341687 & NA & Unknown \\
\hline $\begin{array}{l}\text { S-phase } 2 \text { protein } \\
5 \text { Genes Underexpressed by Subconfluent RPE }\end{array}$ & T69532 & 5.8673496 & NA & Unknown \\
\hline $\begin{array}{l}\text { ATPase, } \mathrm{H}+\text { transporting, lysosomal VO subunit a } \\
\text { isoform } 1\end{array}$ & AA427472 & -6.104330 & -3.6 & $\begin{array}{l}\text { Protein degradation; } \\
\text { Acidification of organelles }\end{array}$ \\
\hline Phosphofructokinase, platelet & R38433 & -6.797924 & -2.4 & Metabolism, glycolysis \\
\hline HIV-1 rev binding protein 2 & W52273 & -7.691879 & -12.2 & Unknown \\
\hline CDNA FU42496 fis, clone BRACE2035003 & AA410207 & -7.320713 & NA & Unknown \\
\hline Solute carrier family 43 , member 3 & N76193 & -6.725273 & -2.2 & Unknown \\
\hline
\end{tabular}

transcription. Native RPE clustered with cells grown in serum withdrawn medium. Since the outer blood-retinal barrier is composed of the RPE and Bruch's membrane, the RPE is probably partially shielded from serum by Bruch's membrane. Our results suggest that serum was a negative factor that separated RPE cells from native cells, and that RPE cells in vivo are not significantly exposed to serum.

Our interpretations are based on a small collection of eyes. The statistical precision of the arrays, however, enabled us to make statistically valid comparisons. While a wide age range was used to reduce age bias, our results may not be generalisable to all native RPE. We were comforted by the validation of our array results by RT-qPCR using a different set of eyes which likely reduces expression differences related to donor to donor variation. We evaluated a single RPE cell line so extrapolation to other cultured RPE cells is unknown. The use of laser microdissection and unamplified RNA for microarray analysis has limitations. While amplification bias 
Table 6 Differential gene expression between cultured RPE cells grown in serum and serum withdrawn conditions sorted by SAM with an FDR of $4.6 \%$ and at least a twofold differential expression threshold

\begin{tabular}{|c|c|c|c|c|}
\hline Gene name & GenBank Acc No & Score (d) & Fold change ${ }^{*}$ & Biological function \\
\hline \multicolumn{5}{|c|}{13 Genes overexpressed by cells grown in serum withdrawn medium } \\
\hline Myeloid cell leukaemia sequence I (BCL2 related) & AA488674 & 2.87841767 & 11.5 & Apoptosis \\
\hline Phytoceramidase, alkaline & W58013 & 2.60586649 & 2.8 & $\begin{array}{l}\text { Apoptosis/ceramide } \\
\text { metabolism }\end{array}$ \\
\hline Reticulocalbin 1, EF-hand calcium binding domain & AA457719 & 2.67862204 & 3.2 & $\begin{array}{l}\text { Calcium binding/signal } \\
\text { transduction }\end{array}$ \\
\hline Visinin-like 1 & H65066 & 2.60870158 & 8.6 & $\begin{array}{l}\text { Calcium binding/signal } \\
\text { transduction }\end{array}$ \\
\hline Phytanoyl-CoA hydroxylase interacting protein & AA405628 & 3.75618502 & 8.3 & Development/differentiation \\
\hline Leucine rich repeat containing 28 & R95132 & 2.99928312 & 6.9 & Development/differentiation \\
\hline Ephrin-B1 & AA428778 & 2.7292518 & 3.2 & Development/differentiation \\
\hline ATP binding cassette, subfamily C (CFTR/MRP), member 13 & H47929 & 3.63023830 & 6.7 & lon transport \\
\hline $\begin{array}{l}\text { Ubiquitin conjugating enzyme E2D } 3 \text { (UBC4/5 homologue, } \\
\text { yeast) }\end{array}$ & AA017199 & 2.72914196 & 3.8 & Protein degradation \\
\hline RNA binding motif protein 4 & AA456271 & 2.79296662 & 2.9 & RNA processing \\
\hline Chromosome 22 open reading frame 8 & H94903 & 3.71707843 & 6.3 & Unknown \\
\hline IMAGE:3895112 & T95342 & 2.66350001 & 4.7 & Unknown \\
\hline Mesoderm development candidate 2 & AA284495 & 2.79383791 & 2.3 & Unknown \\
\hline \multicolumn{5}{|c|}{2 Genes underexpressed by cells grown in serum withdrawn medium } \\
\hline v-myb myeloblastosis viral oncogene homolog (avian)-like 2 & AA456878 & -3.6013598 & -4.5 & Apoptosis/transcription factor \\
\hline IMAGE:295501 & W23543 & -3.4456702 & -2.4 & Unknown \\
\hline
\end{tabular}

Table 7 Differential gene expression between cultured and native RPE by real time RT-PCR

\begin{tabular}{|c|c|c|c|c|}
\hline Gene name & GenBank Acc No & Fold change, array* & Fold change, RT-PCR* & p Value \\
\hline \multicolumn{5}{|l|}{ Subconfluent $v$ native RPE } \\
\hline SEC13-like 1 ( $S$ cerevisiae) & AA496784 & $0 \dagger$ & -12.5 & 0.040 \\
\hline ATPase, $\mathrm{H}+$ transporting, lysosomal $\mathrm{VO}$ subunit a isoform 1 & AA427472 & -3.6 & -1135 & 0.039 \\
\hline Topoisomerase (DNA) II $\alpha 170 \mathrm{kDa}$ & AA504348 & 16.9 & $\infty \ddagger$ & - \\
\hline \multicolumn{5}{|l|}{ Confluent, serum withdrawn $v$ native RPE } \\
\hline START domain containing 4 , sterol regulated & H11369 & -2.5 & -5.7 & 0.0012 \\
\hline Oxidase (cytochrome c) assembly 1-like & AA598582 & 10.5 & 2.71 & 0.0032 \\
\hline Topoisomerase (DNA) II $\alpha 170$ kDa & AA504348 & 17.4 & $\infty \ddagger$ & - \\
\hline
\end{tabular}

${ }^{*}$ Cultured RPE/native RPE; †0, undetectable in subconfluent ARPE-19 cells; $\neq \infty$, undetectable in native RPE,

is eliminated, RNA quantity prevents a comprehensive validation even with RT-qPCR. The array utilised was not RPE specific, and contains both a relatively limited gene set and uncharacterised genes. With further study, the uncharacterised genes may provide new insights into general or RPE specific functioning. Many investigators use a wide range of culture densities, medium, and duration to study RPE function. Our results provide evidence in support of the long held opinion that culture conditions alter the cellular phenotype and validate the long held assumption that great care must be utilised when extrapolating results of gene expression experiments from cultured to native RPE cells.

\section{ACKNOWLEDGEMENTS}

We thank NDRI and the Sierra Eye and Tissue Bank for donor eyes. Supported by NIH/EY 14005 (JTH), NIH/EY 06473 (LMH), and an unrestricted Research to Prevent Blindness grant to the Wilmer Eye Institute, the Michael Panitch Macular Degeneration Research Fund, and gifts from Aleda Wright, and Rick and Sandy Forsythe. JTH is a recipient of a Clinician Scientist Award from the RPB.

\section{Authors' affiliations}

J Tian, K Ishibashi, J T Handa, Michael Panitch Macular Degeneration Research Laboratory, Wilmer Ophthalmological Institute, Johns Hopkins Medical Institutes, Baltimore, MD, USA

S Honda, S A Boylan, L M Hielmeland, Departments of Ophthalmology and Molecular and Cellular Biology, University of California, Davis, CA, USA

Commercial relationships: none

\section{REFERENCES}

1 Dunn KC, Aotaki-Keen AE, Putkey FR, et al. ARPE-19, a human retinal pigment epithelial cell line with differentiated properties. Exp Eye Res 1996:62:155-69

2 Tian J, Ishibashi K, Handa JT. The expression of native and cultured RPE grown on different matrices. Physiol Genomics 2004;17:170-82.

3 Johnston NL, Cervenak J, Shore AD, et al. Multivariate analysis of RNA levels from postmortem human brains as measured by three different methods of RTPCR. Stanley Neuropathology Consortium. J Neurosci Methods 1997;77:83-92.

4 Barthel LK, Raymond PA. Improved method for obtaining 3-microns cryosections for immunocytochemistry. J Histochem Cytochem 1990;38:1383-8

5 Boylan S, Honda S, Hielmeland LM, et al. An optimized protocol for first strand cDNA synthesis from laser capture microdissected tissue. Lab Invest 2001:81:1167-9.

6 Sgroi DC, Teng S, Robinson G, et al. In vivo gene expression profile analysis of human breast cancer progression. Cancer Res 1999;59:5656-61.

7 Lee ML, Whitmore GA. Power and sample size for DNA microarray studies. Stat Med 2002;21:3543-70.

8 Alizadeh AA, Eisen MB, Davis RE, et al. Distinct types of diffuse large B-cell lymphoma identified by gene expression profiling. Nature 2000;403:503-11.

9 Weigel AL, Handa JT, Hielmeland LM. Microarray analysis of H2O2-, HNE-, or $\mathrm{BH}$-treated ARPE-19 cells. Free Radic Biol Med 2002:33:1419-32.

10 Eisen MB, Spellman PT, Brown PO, et al. Cluster analysis and display of genome-wide expression patterns. Proc Natl Acad Sci USA 1998;95:14863-8.

11 Tusher VG, Tibshirani R, Chu G. Significance analysis of microarrays applied to the ionizing radiation response. Proc Natl Acad Sci USA $2001 ; 98: 5116-21$

12 Simpson DA, Feeney S, Boyle C, et al. Retinal VEGF mRNA measured by SYBR green I fluorescence: a versatile approach to quantitative PCR. Mol Vis 2000;6:178-83.

13 Hwang D, Schmitt WA, Stephanopoulos G. Determination of minimum sample size and discriminatory expression patterns in microarray data. Bioinformatics 2002; 18:1184-93. 